\title{
Polydispersity as a Parameter for Indicating the Thermal Stability of Proteins by Dynamic Light Scattering
}

\author{
Kohei ShIBA, ${ }^{* 1, * 2 \dagger}$ Takuro NiIdome, ${ }^{* 1, * 3, * 4}$ Etsuko KatoH, ${ }^{* 5}$ Hongyu XIANG, ${ }^{* 5}$ Lu Han, ${ }^{* 5}$ \\ Takeshi MorI, ${ }^{* 1, * 3}$ and Yoshiki KATAYAMA $* 1, * 3$ \\ *1 Department of Applied Chemistry, Faculty of Engineering, Kyushu University, 744 Motooka, Nishi, \\ Fukuoka 819-0395, Japan \\ *2 Sysmex Corporation, 4-4-4 Takatsuka-dai, Nishi, Kobe, Hyogo 651-2271, Japan \\ *3 Center for Future Chemistry, Kyushu University, 744 Motooka, Nishi, Fukuoka 819-0395, Japan \\ *4 PRESTO, Japan Science and Technology Corporation, Kawaguchi 332-0012, Japan \\ *5 Division of Plant Research, National Institute of Agrobiological Sciences, 2-1-2 Kannondai, Tsukuba, \\ Ibaraki 305-8602, Japan
}

\begin{abstract}
A physical parameter for predicting the thermal stability of proteins was provided by a new approach using dynamic light scattering (DLS). The relationship between the melting point measured by differential scanning calorimetry (DSC) and the polydispersity of the hydrodynamic diameter determined by DLS analysis was examined. Calmodulin (CaM) and concanavalin A (ConA) were used as model proteins. The melting point measured by DSC, an indicator for thermal stability, increased and the polydispersity decreased on binding of the proteins to specific ligands, suggesting that the polydispersity could be used an indicator to predict thermal stability. In addition, the increase of thermal stability that resulted from forming a complex could be quantified by polydispersity analysis even when the melting point changed only slightly.
\end{abstract}

(Received February 25, 2010; Accepted April 5, 2010; Published June 10, 2010)

\section{Introduction}

With the rise of methods such as X-ray crystallography and nuclear magnetic resonance (NMR) for evaluating protein structure, the complete tertiary structure of a number of proteins has been determined. This development has helped to elucidate the critical relationship between protein conformation and function..$^{1-4}$ Many proteins change their structures and stability on binding to ligands: surface plasmon resonance (SPR) is one of the most promising methods for evaluating such protein-ligand interactions. ${ }^{5-9}$ This method is useful for determination of the binding constant $\left(K_{\mathrm{D}}\right)$, and the binding kinetics of protein-ligand interactions in flow systems on solid surfaces. Other methods have been developed to evaluate the protein-ligand interaction in solution. Isothermal titration calorimetry (ITC) and differential scanning calorimetry (DSC) ${ }^{10-14}$ have been used for evaluating protein function. Such calorimetric techniques measure the energy difference between a protein and its complex with a specific ligand. In the case of ITC, the titration of ligands provides a binding constant by calculating the trend of the energy. ${ }^{10,11}$ DSC gives the melting point of a protein, at which the heat capacity curve reaches the maximum value, ${ }^{12-14}$ and reflects the protein stability. ${ }^{15}$ In the tertiary structure of soluble protein in water, hydrophobic domains are generally located in the interior of the structure. When a protein is heated, the

† To whom correspondence should be addressed.

E-mail: shiba.kouhei@ sysmex.co.jp conformation becomes looser than that at the physiological temperature, and the probability of the hydrophobic domains being on the surface is increased. Finally, intermolecular hydrophobic interaction triggers aggregation of proteins. The melting point measured by DSC is an indicator of a looser conformation; thus the melting point can be used as an index of thermal stability of protein. If a specific ligand binds to a protein, the protein structure is stabilized. Since ITC and DSC detect changes in the thermal energy of proteins after adding the ligand, they consequently provide physicochemical parameters of protein-ligand binding.

DLS analysis is a technique for evaluating the hydrodynamic diameter via the Stokes-Einstein equation, ${ }^{16-20}$ which is a relationship between the diameter of a particle and its diffusion coefficient due to Brownian motion in solution. DLS analysis is used to measure the size of nanoparticles such as gold colloids, pigments, liposomes and other materials. ${ }^{21-23}$ Recently, even proteins, which show weak light scattering intensity, can be studied by DLS measurements because of the improvements in instrumental sensitivity. For example, oligomer formation of proteins was detected by DLS as an increase of their hydrodynamic diameters. ${ }^{24-26}$ In addition, the polydispersity obtained from DLS analysis is often used as a parameter for prediction of formation of protein crystals. ${ }^{27,28}$ It indicates that a certain condition, in which the protein has low polydispersity (less than 20\%), should be favorable for growing protein crystals, because homogeneity of protein structure has advantages for crystal growth. ${ }^{27}$

A protein forms its tertiary structure by several types of 
interactions such as hydrophobic, hydrogen bonding and electrostatic interactions. When thermal energy is applied to a protein, the tertiary structure becomes looser, that is, the protein tends to become unfolded. ${ }^{29}$ The unfolded domains in the protein impart viscous resistance against Brownian motion because of augmentations of their fluctuations, and this motion leads to a more random diffusion coefficient.

In the present study, we examined correlation of the polydispersity with thermal stability of proteins by using calmodulin $(\mathrm{CaM}),{ }^{30-33}$ a calcium binding protein, and concanavalin A (ConA), ${ }^{34-36}$ a carbohydrate binding protein, as model proteins. The polydispersity determined by DLS analysis is believed to be a new parameter for indication of the thermal stability of a protein associated with a conformation change.

\section{Experimental}

\section{Reagents and chemicals}

CaM from bovine testes was expressed by pDEST-trx vector ${ }^{37}$ in the BL21(DE3) (Novagen, Madison, WI), and purified using an $\mathrm{Ni}$ column and gel permeation chromatography. The concentration was determined spectrophotometrically at $280 \mathrm{~nm}$ using the value $1560 \mathrm{M}^{-1} \mathrm{~cm}^{-1}$ for the molar extinction coefficient. CaM was prepared in $20 \mathrm{mM}$ Tris- $\mathrm{HCl}$ with $150 \mathrm{mM} \mathrm{NaCl}$ at $\mathrm{pH}$ 7.5. ConA from Jack bean (Sigma-Aldrich Japan, Tokyo, Japan) was purchased. ConA was prepared in $50 \mathrm{mM}$ sodium 4-(2-hydroxyethyl)-1-piperazineethanesulfonate (HEPES) buffer ( $\mathrm{pH} 7.5$ ); its concentration in the solution was determined spectrophotometrically at $280 \mathrm{~nm}$ using the value $26030 \mathrm{M}^{-1} \mathrm{~cm}^{-1}$ for the molar extinction coefficient. All samples were filtered using $0.22 \mu \mathrm{m}$ nylon filters. The molar extinction coefficients were calculated from the numbers of tryptophan, tyrosine and cystine residues in the protein. ${ }^{38}$ $d$-Galactose, $d$-glucose and $d$-mannose (Sigma-Aldrich Japan, Tokyo, Japan) were used without further purification. All other reagents used were analytical quality, and all aqueous solutions were prepared with Milli-Q water on a Millipore water purification system.

\section{DSC measurement}

DSC $^{15}$ was carried out using a VPcapillary DSC platform (MicroCal, Northamption, MA). The concentration of CaM was $2.0 \mathrm{mg} / \mathrm{mL}$, equivalent to $117 \mu \mathrm{M}$ as a monomer. CaM binds to up to four $\mathrm{Ca}^{2+}$ ions. We prepared solutions of $\mathrm{CaM}$ in $20 \mathrm{mM}$ Tris- $\mathrm{HCl}$ with $150 \mathrm{mM} \mathrm{NaCl}$, at $\mathrm{pH}$ 7.5, with $0,117,235,471$, 941 and $2353 \mu \mathrm{M}$ of $\mathrm{CaCl}_{2}$. The measurements were performed at temperatures from 15 to $150^{\circ} \mathrm{C}$ at scan rate of $1.0^{\circ} \mathrm{C} / \mathrm{min}$. The concentration of ConA was $2.0 \mathrm{mg} / \mathrm{mL}$, equivalent to $80 \mu \mathrm{M}$ as a monomer. The concentration of each of various carbohydrate solutions was $1 \mathrm{mM}$. Protein samples were mixed with the same volume of various carbohydrates in the same buffer, such that the total amount of carbohydrates was sufficient for binding in the carbohydrate recognition domain of ConA. The measurements were performed at temperatures from 15 to $65^{\circ} \mathrm{C}$ at a scan rate of $1.0^{\circ} \mathrm{C} / \mathrm{min}$. All samples were degassed before measurements. The melting point was determined as the temperature at which the heat capacity curve reached the maximum value. ${ }^{15}$

Calculation of the distribution of various proteins from DLS measurements

Size distributions were measured with a Zetasizer NanoZS (Malvern Instruments, UK), ${ }^{39}$ based on DLS. The particle size distribution from DLS measurement was derived by deconvolution of the measured intensity autocorrelation function of the sample. ${ }^{40}$ Generally, deconvolution was accomplished using a non-negatively constrained least squares (NNLS) ${ }^{41,42}$ fitting algorithm, common examples being CONTIN, ${ }^{43}$ Regularization, and the General Purpose and Multiple Narrow Mode algorithms ${ }^{44,45}$ included in the Zetasizer Nano software. The distribution was obtained by the NNLS method using an alpha value, which is a function parameter of the fitting sensitivity of the raw data. ${ }^{46}$ We used a fixed alpha value to measure the polydispersity. All of distributions were calculated by the value of 0.01 . The CaM and ConA samples used for DLS analysis were the same preparations as those that were used for DSC measurements. All samples were measured at $20^{\circ} \mathrm{C}$ and all results were evaluated as the mean values of five replicates. In addition, the temperature trend of DLS measurements was determined at $1{ }^{\circ} \mathrm{C}$ intervals from 15 to $50^{\circ} \mathrm{C}$. The polydispersity (Pd) was evaluated according to Eq. (1), where SD is the standard deviation of the distribution and $\mathrm{D}(\mathrm{H})$ is the mean hydrodynamic diameter from DLS measurement:

$$
\mathrm{Pd}=\frac{\mathrm{SD}}{\mathrm{D}(\mathrm{H})} \times 100(\%)
$$

\section{Results and Discussion}

\section{Change of the polydispersity of CaM by binding with calcium} ions

The polydispersity evaluated using Eq. (1) meant the variability of the DLS distribution. First, we selected CaM as a model protein. $\mathrm{CaM}$ is known to bind calcium ions and dramatically changes structure from the apo- to the holo-form..$^{30}$ The binding of calcium ion induces not only a structural change of CaM but also a change in its melting point as determined by DSC measurement. ${ }^{30,31}$ Figure 1 shows DSC scans of apo- and holo-CaM; the DSC data agreed with results previously reported. ${ }^{30}$ This result indicated that the holo-form was stabilized by binding of calcium ions that induced a conformational change of CaM from the apo-form. Figure 2 shows the size distributions of apo- and holo-CaM. While no significant difference between the mean hydrodynamic diameters of apo- and holo-CaM was detected, the widths of the size distribution, reflecting the polydispersities, were different. The polydispersity of holo-CaM was about $23 \%$, and smaller than that of apo-CaM (31\%; data not shown).

The reason for the increase of the melting point from apo- to holo-form is that this domain becomes stiffer upon binding calcium ions. In the apo-form, CaM has a hinge site in the middle of the whole sequence. Three dimensional structures of apo- ${ }^{32}$ and holo-CaM, ${ }^{33}$ from the Protein Data Bank (PDB), are shown in Fig. 3. When four calcium ions bind to a $\mathrm{CaM}$ molecule, the middle part transforms from a random loop to an $\alpha$-helix. Since this part of CaM changed to the stiffer holo-form, we suppose that the fluctuation of the main chain of the protein was restricted. When the fluctuation of the main backbone was restricted, the heterogeneity of Brownian motion might be limited by restricted fluctuation of the backbone. Brownian motion is evaluated by DLS analysis as a diffusion coefficient parameter. Since DLS measures diffusion coefficient, the polydispersity of diffusion coefficient was decreased, corresponding to decreased polydispersity of the hydrodynamic diameter measured by DLS. Considering the results from DLS analysis and DSC, we conclude that the polydispersity is related to the melting point of $\mathrm{CaM}$.

To confirm the relationship between the melting point and 


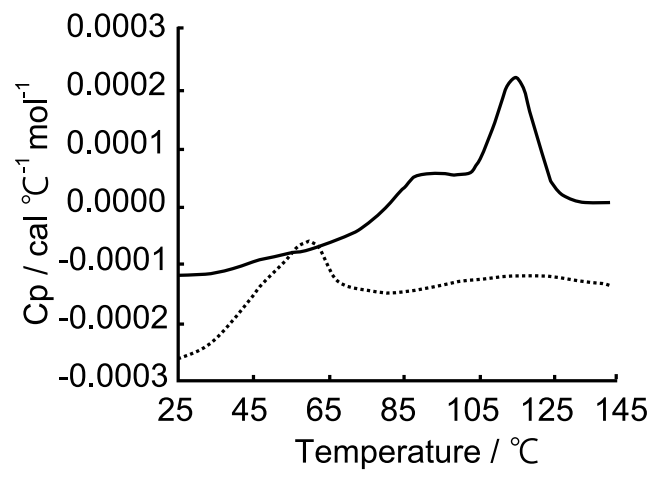

Fig. 1 Temperature dependence of the partial molar heat capacity of $\mathrm{CaM}$ alone (apo-form, dotted line) and of $\mathrm{Ca}^{2+}-\mathrm{CaM}$ complex (holo-form, solid line).

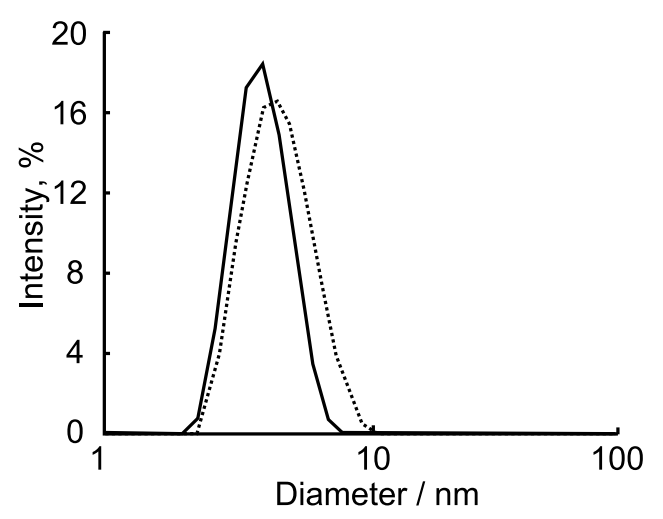

Fig. 2 DLS size distribution of CaM alone (apo-form, dotted line) and $\mathrm{Ca}^{2+}+\mathrm{CaM}$ complex (holo-form, solid line).

polydispersity of $\mathrm{CaM}$, we added calcium ions to $\mathrm{CaM}$ at several $\mathrm{Ca} / \mathrm{CaM}$ ratios (1, 2, 4, 8 and 20); then the melting points were determined by DSC and the polydispersities by DLS (Fig. 4). The titration with calcium ions demonstrated that the melting point of $\mathrm{CaM}$ increased with increase of the ratio of calcium ions to $\mathrm{CaM}$ molecules. At the half-stoichiometric ratio (two calcium ions per $\mathrm{CaM}$ ), the melting point of $\mathrm{CaM}$ was $97^{\circ} \mathrm{C}$, thus $38^{\circ} \mathrm{C}$ higher than for apo-CaM. For the stoichiometric ratio (four calcium ions per $\mathrm{CaM}$ ), the melting point of $\mathrm{CaM}$ was increased to $104^{\circ} \mathrm{C}$, and over a further four-fold range of $\mathrm{Ca} / \mathrm{CaM}$ ratios the melting point reached a plateau value. The polydispersity of apo-CaM was $31 \%$, and it decreased on increasing the ratio of calcium ions to CaM. For the sample with $\mathrm{Ca} / \mathrm{CaM}$ ratio $=20$, the polydispersity decreased to $23 \%$. Thus, the polydispersity of $\mathrm{CaM}$ showed an inverse correlation with its melting point.

Change of the polydispersity of ConA by addition of carbohydrates Figure 3 shows that the structure of CaM changed dramatically by addition of calcium ions. Such change was easily measured by DLS as a change of polydispersity corresponding to change in the extent of fluctuation of the molecular main backbone. Next, we attempted to measure another protein, ConA, whose structure changes little with binding to ligands. ${ }^{34}$ It is known from PDB information that specific carbohydrates can bind to the surface of ConA. In particular, ConA can bind four $\alpha-1,2$ mannobiose molecules (data not shown). ${ }^{35}$ We studied
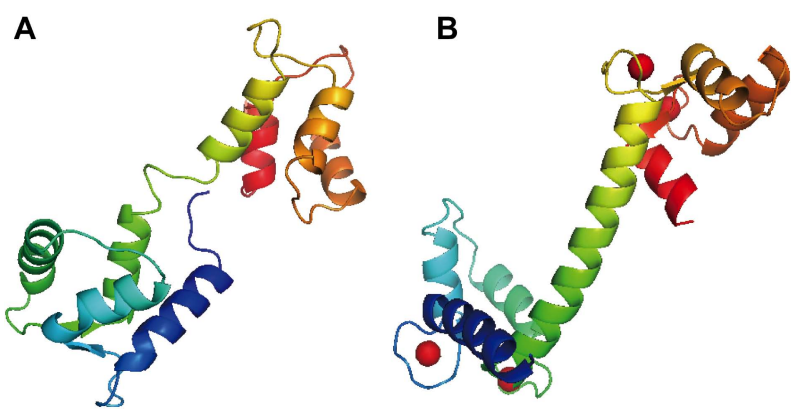

Fig. 3 Three-dimensional structure of CaM (apo-form, panel A) and $\mathrm{Ca}^{2+}-\mathrm{CaM}$ complex (holo-form, panel B). The PDB IDs of apo-CaM and holo-CaM are 1CFC and 1CLL, respectively.

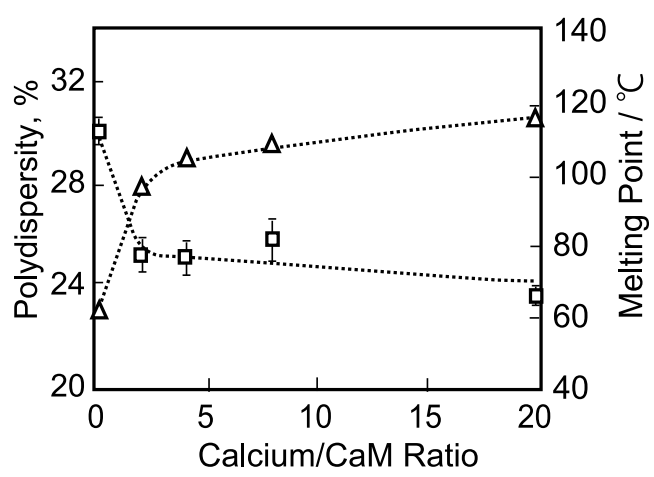

Fig. 4 Trends of the polydispersity and the melting point for CaM titrated with calcium ion. The squares indicate polydispersity values (left $\mathrm{Y}$ axis) and the triangles represent melting point (right axis).

complexes of ConA with various carbohydrates by DSC and DLS analysis. Figure 5 shows DSC scans of ConA and ConA-mannose complex. The polydispersity of the complex was significantly smaller than that of free ConA (Fig. 6), while the melting point of the complex $\left(61.7^{\circ} \mathrm{C}\right)$ was slightly higher than that of free ConA $\left(60.9^{\circ} \mathrm{C}\right)$. This result indicates that DLS analysis of ConA is advantageous for detecting its carbohydrate binding clearly compared with DSC. In Table 1, we summarize the melting points, polydispersities and dissociation constants, $K_{1}$, of ConA with several carbohydrates evaluated by frontal affinity chromatography (FAC) analysis. ${ }^{47,48}$ DLS analysis and FAC clearly showed that ConA had higher affinity to mannose and glucose than to galactose. ${ }^{36}$

It is thought that the small difference of the melting points before and after binding of carbohydrate was caused by a small difference of the ConA structure between its apo- and holo-forms due to binding of the carbohydrate to the protein surface. To predict the difference of the thermal stabilities of ConA in the presence and absence of carbohydrate, DLS analysis of ConA and ConA-mannose complex was performed at various temperatures. Figure 7 shows the temperature dependence of hydrodynamic diameter for ConA and the ConA-mannose complex. Sharply increasing hydrodynamic diameter at a particular temperature corresponds to the onset of aggregation of ConA and its complex with mannose. From 15 to $35^{\circ} \mathrm{C}$, the hydrodynamic diameter remained the same as the initial value and indicated that no aggregation was occurring. The hydrodynamic diameter of ConA started to increase at $42^{\circ} \mathrm{C}$, and above $42^{\circ} \mathrm{C}$ became very large by aggregation. However, 


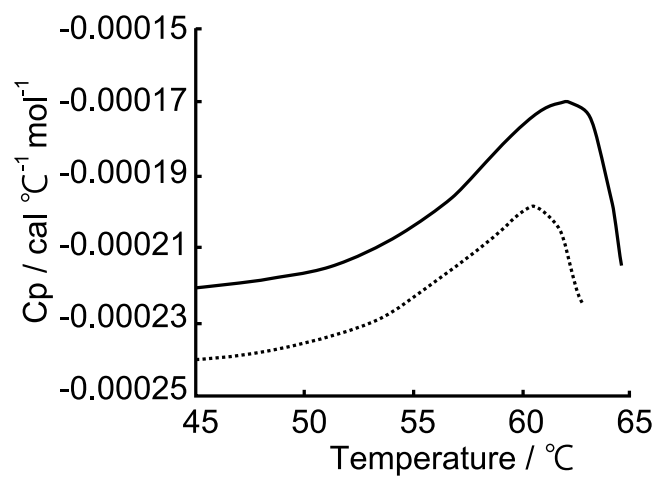

Fig. 5 Temperature dependence of the partial molar heat capacity of ConA alone (dotted line) and ConA-mannose complex (solid line).

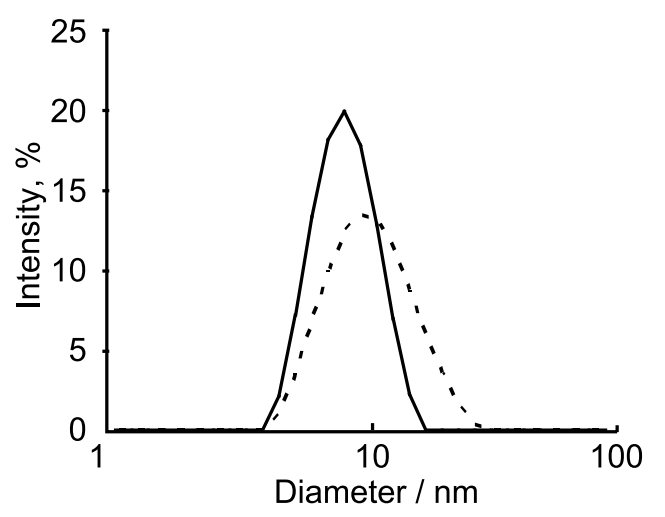

Fig. 6 DLS size distribution of ConA alone (dotted line) and ConA-mannose complex (solid line).

the hydrodynamic diameter of ConA-mannose complex started to increase at $47^{\circ} \mathrm{C}$, indicating aggregation above a significantly higher temperature. These observations are very interesting, because the denaturing temperature, a melting point in DSC analysis, was only slightly changed by mannose binding. ${ }^{15}$ Thus the critical temperature for ConA aggregation was increased by complexation with mannose. The difference of the critical temperatures should be related to the thermal stability of ConA. That suggested that the probability of hydrophobic domains appearing on the surface was reduced due to decreased fluctuation of ConA surface by formation of the complex with mannose, and the critical temperature was increased after formation of the complex. Even though the increase of the melting point was very small, the thermal stability was enhanced by forming a complex with specific carbohydrates.

When four carbohydrates bound to the ConA surface, the polydispersity was also reduced corresponding to the affinity. The change in polydispersity indicates a difference between the surfaces of ConA and ConA-carbohydrate complex. We expected that the heterogeneity of Brownian motion, which originates from the variety of protein structures, was limited by restricted fluctuations of the surface corresponding to complex formation. Hence, it is suggested that the polydispersity is an indicator of the thermal stability of proteins with specific ligands.
Table 1 Experimental data for ConA-carbohydrate interaction

\begin{tabular}{lccc}
\hline \multicolumn{1}{c}{ Analyte } & $T_{\mathrm{m}}{ }^{\mathrm{a}}{ }^{\circ} \mathrm{C}$ & $\mathrm{Pd}^{\mathrm{b}}, \%$ & $K_{1} \mathrm{c} / \mathrm{mM}$ \\
\hline ConA & 60.9 & $40.5 \pm 0.9$ & N.A. \\
ConA-galactose complex & 61.1 & $40.3 \pm 1.0$ & 260 \\
ConA-glucose complex & 61.6 & $34.0 \pm 2.2$ & 1.5 \\
ConA-mannose complex & 61.7 & $29.2 \pm 0.6$ & 0.19 \\
\hline
\end{tabular}

N.A.: not applicable.

a. $T_{\mathrm{m}}$ is the melting point obtained by DSC.

b. $\mathrm{Pd}$ is the polydispersity of the mean hydrodynamic diameter from five replicated DLS measurements.

c. $K_{1}$ is the dissociation constant from FAC.

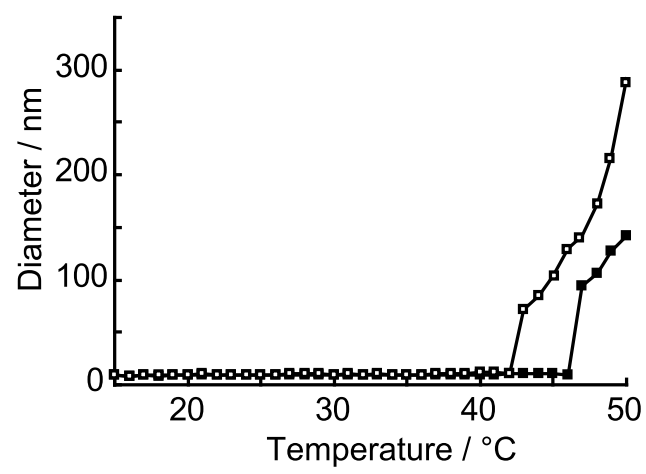

Fig. 7 Temperature trends of the hydrodynamic diameter of ConA and its mannose complex. The open squares indicate the hydrodynamic diameter of ConA, and the shaded squares refer to the ConA-mannose complex.

\section{Conclusion}

In this study, we found that binding of a specific ligand to a protein induced a decrease of the DLS polydispersity of the protein. When the ligand binds to the protein, fluctuation of the main chain and the surface structure are restricted, and the heterogeneity of Brownian motion, which originates from the variety of protein structures, was limited by restricted fluctuations of their structure.

The restriction of the fluctuations of the protein molecule was expected to be reflected in a change of the melting point determined by DSC. In the case of CaM, the polydispersity was decreased 30 to $23 \%$ by binding calcium ions corresponding to an increase of the melting point. For ConA, the polydispersity was also decreased by forming a complex with carbohydrates, related to the affinity and corresponding to thermal stability. Since the melting point is used as an indicator for the thermal stability of proteins, we suggest that there is a relationship between DLS polydispersity and thermal stability of proteins.

Understanding protein functions in relation to their structures is important not only for revealing molecular mechanism in biology but also for drug development. As the development of protein drugs goes forward to the industrial phase, tests of stability and functionality in solution are necessary. DLS analysis is more rapid than current assay methods such as ITC and DSC, so it might be effective as a high throughput assay. Thus the polydispersity obtained from DLS measurement will be a powerful tool for predicting the thermal stability of proteins and their functions. 


\section{Acknowledgements}

This research was supported by a Grant-in-Aid for Scientific Research (B) (No. 19300172) from the Japan Society for the Promotion of Science. We thank Dr. Ulf Nobbmann, Malvern Instruments, LTD., for helpful advice concerning the DLS instrument.

\section{References}

1. F. I. Andersson, A. Tryggvesson, M. Sharon, A. V. Diemand, M. Classen, C. Best, R. Schmidt, J. Schelin, T. M. Stanne, B. Bukau, C. V. Robinson, S. Witt, A. Mogk, and A. K. Clarke, J. Biol. Chem., 2009, 284, 13519.

2. A. K. Meinild, D. D. F. Loo, S. Skovstrup, U. Gether, and N. MacAulay, J. Biol. Chem., 2009, 284, 16226.

3. S. R. C. Clemont, C. A. French, N. C. Kaarsholm, and M. F. Dunn, Biochemistry, 1997, 36, 5837.

4. T. Stromer, M. Ehrnsperger, M. Gaestel, and J. Buchner, J. Biol. Chem., 2003, 278, 18015.

5. A. Frostell-Karlsson, A. Remaeus, H. Roos, K. Andersson, P. Borg, M. Hamalainen, and R. Karlsson, J. Med. Chem., 2000, 43, 1986.

6. D. G. Myszka and R. L. Rich, Pharm. Sci. Technol. Today, 2000, 3, 310.

7. R. L. Rich, Y. S. N. Day, T. A. Morton, and D. G. Myszka, Anal. Biochem., 2001, 296, 197.

8. D. G. Myszka, Anal. Biochem., 2004, 329, 316.

9. P. Sandblad, J. Arnell, and T. Fornstedt, Anal. Chem., 2009, 81,3551 .

10. M. Osawa, A. Dace, K. I. Tong, A. Valiveti, and M. Ikura, J. Biol. Chem., 2005, 280, 18008.

11. R. S. Hodges, J. Mills, S. McReynolds, J. P. Kirwan, B. Tripet, and D. Osguthorpe, J. Mol. Biol., 2009, 392, 747.

12. J. F. Brandts and L. N. Lin, Biochemistry, 1990, 29, 6927.

13. M. J. Waner, I. Navrotskaya, A. Bain, E. D. Oldham, and D. P. Mascotti, Biophys. J., 2004, 87, 2701.

14. F. P. Schwerz, H. Ahmed, M. A. Bianchet, L. M. Amzel, and G. R. Vasta, Biochemistry., 1998, 37, 5867.

15. B. D. Ladbrooke, R. M. Williams, and D. Chapman, Biochem. Biophys. Acta, 1968, 150, 333.

16. W. Burchard, M. Schmidt, and W. H. Stockmayer, Macromolecules, 1980, 13, 1265.

17. G. D. Phillies, Anal. Chem., 1990, 62, 1049A.

18. N. C. Santos and M. A. Castanho, Biophys. J., 1996, 71, 1641.

19. D. Lochmann, J. Weyermann, C. Georgens, R. Prassl, and A. Zimmer, Eur. J. Pharm. Biopharm., 2005, 59, 419.

20. M. Rousselot, E. Jaenicke, T. Lamkemeyer, J. R. Harris, and R. Pirow, FEBS Journal, 2006, 273, 4055.

21. S. Wang and L. Gao, J. Mat. Sci. Lett., 1999, 18, 1891.
22. P. R. Dash, M. L. Read, K. D. Fisher, K. A. Howard, M. Wolfert, D. Oupicky, V. Subr, J. Strohalm, K. Ulbrich, and L. W. Seymour, J. Biol. Chem., 2000, 275, 3793.

23. S. Kawakami, Y. Ito, S. Fumoto, F. Yamashita, and M. Hashida, J. Gene. Med., 2005, 7, 1526.

24. G. Plakoutsi, N. Taddei, M. Stefani, and F. Chiti, J. Biol. Chem., 2004, 279, 14111.

25. C. Le Bon, T. Nicolai, and D. Durand, Int. J. Food Sci. Technol., 1999, 34, 451.

26. J. Moradian-Oldak, W. Leung, and A. G. Fincham, J. Struct. Biol., 1998, 122, 320.

27. B. E. Bernstein, P. A. M. Michels, H. Kim, P. Petra, and W. G. J. Hol, Protein Sci., 1998, 7, 504.

28. J. D. Phillips, F. G. Whitby, J. P. Kusher, and C. P. Hill, Protein Sci., 1997, 6, 1343.

29. O. Jardetzky and N. G. Wade-Jardetzky, Annu. Rev. Biochem., 1971, 40, 605.

30. P. L. Wintrode and P. L. Privalov, J. Mol. Biol., 1997, 266, 1050.

31. A. Crivici and M. Ikura, Annu. Rev. Biophys. Biomol. Struct., 1995, 24, 85.

32. H. Kuboniwa, N. Tjandra, S. Grzesiek, H. Ren, C. B. Klee, and A. Bax, Nat. Struct. Biol., 1995, 2, 768.

33. R. Chattopadhyaya, W. E. Meador, A. R. Means, and F. A. Quiocho, J. Mol. Biol., 1992, 228, 1177.

34. J. Bouckaert, F. Poortmans, L. Wyns, and R. Loris, J. Biol. Chem., 1996, 271, 16144.

35. D. N. Moothoo, B. Canan, R. A. Field, and J. H. Naismith, Glycobiology, 1999, 9, 539.

36. M. S. Hargrove, D. Barrick, and J. S. Olson, Biochemistry, 1996, 35, 11293.

37. Y. Tsunoda, N. Sakai, K. Kikuchi, S. Katoh, K. Akagi, J. Miura-Ohnuma, Y. Tashiro, K. Murata, N. Shibuya, and E. Katoh, Protein Expression Purif., 2005, 42, 268.

38. S. C. Gill and P. H. von Hippel, Anal. Biochem., 1989, 182, 319.

39. P. L. Privalov and N. N. Khechinashvili, J. Mol. Biol., 1974, 86, 665.

40. K. Mattison and M. Kaszuba, Am. Biotechnol. Lab., 2003, June, 28.

41. J. D. Morrison, J. D. Corcoran, and K. E. Lewis, J. Appl. Crystallogr., 1992, 25, 504.

42. S. W. Provencher, Macromol. Chem., 1979, 180, 201.

43. N. Ostrowsky, D. Sornette, P. Parker, and E. R. Pike, Optica Acta, 1981, 28, 1059.

44. S. W. Provencher, Comput. Phys. Commun., 1982, 27, 229.

45. K. Mattison and J. Jones, Biopharm. Int., 2001, 14, 56.

46. I. D. Morrison and E. F. Grabowski, Langmuir, 1985, 1, 496.

47. Y. Oda, K. Kasai, and S. Ishii, J. Biochem., 1981, 89, 285.

48. Y. Ohyama, K. Kasai, H. Nomoto, and Y. Inoue, J. Biol. Chem., 1985, 260, 6882. 